\title{
A computationally efficient model to capture the inertia of the piezoelectric stack in impact drive mechanism in the case of the in-pipe inspection application
}

\author{
Jin $\mathrm{Li}^{1}$, Chang Jun Liu ${ }^{1}$, Xin Wen Xiong ${ }^{1}$, Yi Fan Liu ${ }^{2}$, and Wen Jun Zhang ${ }^{3}$ \\ ${ }^{1}$ The Complex and Intelligent System Research Center, School of Mechanical and Power Engineering, \\ East China University of Science and Technology, Meilong Road 130, Shanghai, 200237, China \\ ${ }^{2}$ Robotic Systems Laboratory, Ecole Polytechnique Fédérale de Lausanne (EPFL), C/o Nicolas Cantale Avenue \\ de prefaully 56, 1020 Lausanne, Switzerland \\ ${ }^{3}$ Department of Mechanical Engineering, University of Saskatchewan, Saskatoon, S7N5A9, Canada
}

Correspondence to: Chang Jun Liu (cjliu@ecust.edu.cn)

Received: 21 October 2015 - Revised: 28 January 2016 - Accepted: 7 March 2016 - Published: 17 March 2016

Abstract. This paper presents a new model for the piezoelectric actuator (PA) in the context of in the impact drive mechanism (IDM) for the in-pipe inspection application. The feature of the model is capturing the inertia of PA stack in a distributed manner as opposed to the lumped manner in literature. The benefit arising from this feature is a balanced trade-off between computational efficiency and model accuracy. The study presented in this paper included both theoretical development (i.e. the model of the piezoelectric actuator and the model of the entire IDM which includes the actuator) and experimental verification of the model. The study has shown that (1) the inertia of the PA in such a robot will significantly affect the accuracy of the entire model of IDM and (2) the simulation of the dynamic behavior with the proposed model is sufficiently accurate by comparing with the experiment. It is thus recommended that the inertia of the PA be considered in the entire model of the IDM robot. The model is an analytical type, which has a high potential to be used for the model-based control of the IDM robot and optimization of its design for a much improved performance of the IDM system.

A tremendous amount of progresses in micro-robotics have been made in recent years, including micromanipulation for micro or nano-objects handling and nano-positioning. These systems have many applications ranging from in vivo biomedical therapeutic procedures to military reconnaissance (Fatikow and Rembold, 2009; Ouyang et al., 2008). In-pipe robots driven by piezoelectric actuators (PA) have advantages of low energy consumption, small size, and high precision down to the scale of micrometers and even nanometers. In-pipe robots can perform specific functions (scanning, detecting and repairing) in small pipes, such as condenser pipe, blood vessel and intestine (Dario et al., 2003; Yukawa et al., 2006; Xia et al., 2008).

One such type of actuators is based on the stick-slip (S-S) actuation principle (Zhang et al., 2012). In order to realize the autonomous motion, one type of the S-S actuator called impact drive mechanism (IDM) (Dario et al., 2003; Tenzer and Mrad, 2004; Fukui et al., 2001) was proven to be very useful. The schematic diagram of IDM is shown in Fig. 1. The system consists of a main body, an attached block, and a PA (e.g. PZT actuator). Take the motion towards right as a forward motion. The forward motion has three stages: (1) neutral state at which the PA is given the zero voltage; (2) slow extension at which the voltage increases slowly and thus the PA extends slowly to push the block with a small acceleration (note that the main body holds its position by the static friction force from the in-wall of the pipe); (3) quick contraction at which the driving voltage suddenly drops down to zero, and the PA contracts quickly and pulls the main body moving forward. By repeating the foregoing actions, the main body is moved forward continuously (step by step; like a step motor). There is possibility that PA is replaced by other direct 


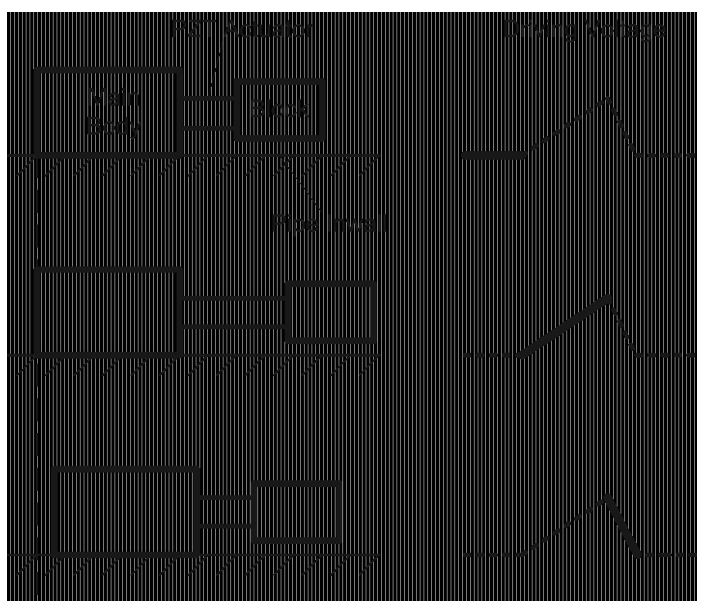

Figure 1. The working principle of the IDM robot.

driven actuators such as shape memory alloy (Hattori et al., 2014). Further, the special requirement on the input voltage (i.e. with one direction of the stroke fast and the opposite direction of the stroke slow) can be realized by the resonant principle (Yokozawa and Morita, 2015).

A model that describes the dynamics of the IDM system is useful to the control of the system and to optimization of the structure of the system, including both the mechanism and controller (Zhang et al., 1999; Li et al., 2001; Liu et al., 2015). Liu et al. (2009) studied the IDM robot actuated by a piezoelectric bimorph (which is of a different configuration of piezoelectric materials to generate motion or deformation) based on a model without consideration of the inertia of the PA. Makkar et al. (2007) and Li et al. (2009) studied the modeling for friction without consideration of the inertia of PA. Ha et al. (2005) optimized the mass ratio of the block and main body and the driving waveform (Fig. 1); however they have not included the inertia of PA in their analysis. Hunstig et al. (2013) investigated the performance and the velocity limitation of the piezoelectric inertia drives under ideal excitations. Sabzehmeidani et al. (2010) studied IDM intelligent control based on mass-spring-damping model with 2 DOFs. Their model has not explicitly included the dynamics of the PA.

In the previous studies where the inertia of the PA is considered, the PA was usually treated as an ideal spring and damping with zero mass (Higuchi et al., 1993; Chang and Li, 1999). However, the inertia of the PAs consumes the driving power and finally influences the dynamic behaviors of the robot. Their effects may be comparable with those out of other components. This means that consideration of the inertia of PA especially in the form of a continuous media may be necessary especially for micro-systems (Liu et al., 2015). Further, aiming at the control of such a system, an analytical model with reasonable accuracy is highly useful (Yokose et al., 2014).

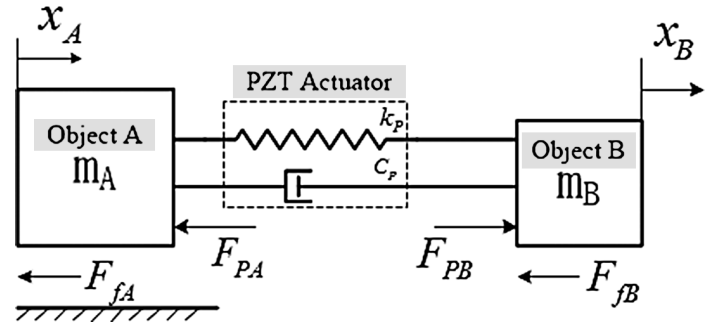

Figure 2. The free-body diagram of the IDM robot.

This paper presents a study on developing an analytical model for the IDM robot in the context of in-pipe inspection. The inertia of PA and that of the other components in this case are comparable. The experiment was carried out to verify the effectiveness of the model. The remainder of the paper is organized in the following. Section 2 presents the model. Section 3 discusses the experimental verification. Section 4 presents some model-predicted results behind this IDM inpipe inspection robot, which are useful to optimizing the design of the IDM in-pipe robot. Finally, there is a conclusion in Sect. 5.

\section{Model development}

\subsection{Governing equation}

The free body diagram of the IDM in-pipe robot is shown in Fig. 2. The entire system moves along the $x$ direction, where $m_{\mathrm{A}}$ and $m_{\mathrm{B}}$ represent the mass of the main body (Object A) and the attached block (Object B), and $x_{\mathrm{A}}$ and $x_{\mathrm{B}}$ are the corresponding displacements. From Fig. 2 and by applying the Newtonian second law, one can obtain the following two equations on Objects A and B, respectively

$m_{\mathrm{A}} \ddot{x}_{\mathrm{A}}=k_{P}\left(x_{\mathrm{B}}-x_{\mathrm{A}}\right)+c_{p}\left(\dot{x}_{\mathrm{B}}-\dot{x}_{\mathrm{A}}\right)-F_{\mathrm{fA}}-F_{\mathrm{PA}}$
$m_{\mathrm{B}} \ddot{x}_{\mathrm{B}}=-k_{P}\left(x_{\mathrm{B}}-x_{\mathrm{A}}\right)-c_{p}\left(\dot{x}_{\mathrm{B}}-\dot{x}_{\mathrm{A}}\right)-F_{\mathrm{fB}}+F_{\mathrm{PB}}$.

In the above equations, the stiffness and damping of the PA are expressed as $k_{P}$ and $c_{p}$, respectively. $F_{\mathrm{fA}}$ and $F_{\mathrm{fB}}$ are the friction forces acting on the main body and block, respectively. $F_{\mathrm{PA}}$ and $F_{\mathrm{PB}}$ are the driving forces generated from the PA, respectively. It is noted that $F_{\mathrm{PA}} \neq F_{\mathrm{PB}}$ (because the dynamics of the PA is considered). Further, the in-pipe inspection robot here has revised a little bit on the IDM, that is, having the inertial mass (i.e. block B) down to the ground.

\subsection{Dynamic model of the PA}

The PA is in itself a dynamics system, which means that the PA has inertia, damping, and stiffness. Suppose the PA is an elastic body and its inertia is uniformly distributed along the $x$ direction. Under the configuration as shown in Fig. 1, the piezoelectric rod extends such that Object A tends to move 
along the left direction while Object B tends to move along the right direction. As such, there must be an instantaneous center of velocity at a certain time during contraction or at a certain position along the piezoelectric rod. We cut the whole PA rod into two segments on this instantaneous center. Figure 3 shows two separate force diagram of these two parts of the PA, where $F_{P}$ is the driving force generated in the PA rod by the input voltage, $d l_{\mathrm{A}}$ and $\mathrm{dl}_{\mathrm{B}}$ are the displacements of the two ends of the whole PA rod during the time period of $d t$, at which the instantaneous center forms, $m_{\mathrm{PA}}$ and $m_{\mathrm{PB}}$ are the masses of the two parts of the PA rod, respectively.

The works with the two parts of the PA rod during $d t$ are:

$$
\begin{aligned}
& d W_{\mathrm{A}}=\left(F_{\mathrm{PA}}-F_{\mathrm{P}}\right) d l_{\mathrm{A}}=\left(F_{\mathrm{PA}}-F_{P}\right)\left(v_{\mathrm{A}} d t+\frac{1}{2} a_{\mathrm{A}} d t^{2}\right) \\
& d W_{\mathrm{B}}=\left(F_{P}-F_{\mathrm{PB}}\right) d l_{\mathrm{B}}=\left(F_{P}-F_{\mathrm{PB}}\right)\left(v_{\mathrm{B}} d t+\frac{1}{2} a_{\mathrm{B}} d t^{2}\right) .
\end{aligned}
$$

The kinetic energy of $m_{\mathrm{PA}}$ at a certain time is:

$$
E_{\mathrm{PA}}=\int_{0}^{l} \frac{1}{2} v_{x}^{2} \rho d x=\int_{0}^{l} \frac{1}{2}\left(\frac{x}{l} v\right)^{2} \rho d x=\frac{1}{6} m v^{2} .
$$

The change in the kinetic energy within $d t$ is:

$$
\begin{aligned}
d E_{\mathrm{PA}} & =\frac{1}{6} m_{\mathrm{PA}}\left[\left(v_{\mathrm{A}}+a_{\mathrm{A}} d t\right)^{2}-v_{A}^{2}\right] \\
& =\frac{1}{6} m_{\mathrm{PA}} a_{\mathrm{A}}^{2} d t^{2}+\frac{1}{3} m_{\mathrm{PA}} v_{\mathrm{A}} a_{\mathrm{A}} d t .
\end{aligned}
$$

Note that the change in kinetic energy within $d t$ should be equal to the work done during this time. That is,

$d E_{\mathrm{PA}}=d W_{\mathrm{A}}$.

From Eqs. (3), (6), (7), we have (for the left part of the piezoelectric rod P1):

$F_{\mathrm{PA}}=\frac{1}{3} m_{\mathrm{PA}} a_{\mathrm{A}}+F_{P}=\frac{1}{3}\left(\frac{\dot{x}_{\mathrm{A}}}{\dot{x}_{\mathrm{A}}-\dot{x}_{\mathrm{B}}}\right) m_{P} \ddot{x}_{\mathrm{A}}+F_{P}$.

Similarly, we have (for the right part of the piezoelectric $\operatorname{rod} \mathrm{P} 2)$ :

$F_{\mathrm{PB}}=-\frac{1}{3} m_{\mathrm{PB}} a_{\mathrm{B}}+F_{P}=\frac{1}{3}\left(\frac{\dot{x}_{\mathrm{B}}}{\dot{x}_{\mathrm{A}}-\dot{x}_{\mathrm{B}}}\right) m_{P} \ddot{x}_{\mathrm{B}}+F_{P}$.

\subsection{Friction model}

Without loss of the generality for the purpose of this study, the Karnopp model (Karnopp, 1985) was employed to describe the frictional force in this work, i.e.:

$F_{\mathrm{f}}=\left\{F_{\mathrm{C}} \lambda(v)+F_{\mathrm{S}}[1-\lambda(v)]\right\} \operatorname{sign}(v)$
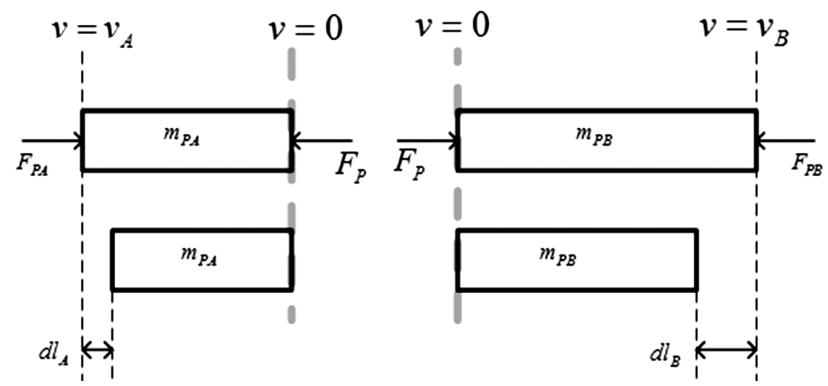

Figure 3. The free-body diagram of the PZT actuator.

where

$\lambda(v)=\left\{\begin{array}{ll}1 & |v|>\alpha \\ 0 & |v| \leq \alpha\end{array} \alpha>0\right.$.

$F_{\mathrm{C}}=\mu_{K} F_{\mathrm{n}}$ is the Coulomb friction, and $F_{\mathrm{S}}=\mu_{S} F_{\mathrm{n}}$ is the static friction. $F_{\mathrm{n}}$ denotes the normal force, $\mu_{K}$ and $\mu_{S}$ are the frictional coefficients, respectively. It is noted that there is a friction force on Object $\mathrm{A}$ and Object $\mathrm{B}$, respectively.

\subsection{Model integration}

The ultimate goal of the analysis is to find the motion of the main body (Object A) and the inertial mass or block (Object B), as shown in Figs. 1 and 2. For the convenience of the reader, we put together all the equations as derived before in the following.

$$
\left\{\begin{array}{l}
m_{\mathrm{A}} \ddot{x}_{\mathrm{A}}=k_{P}\left(x_{\mathrm{B}}-x_{\mathrm{A}}\right)+c_{p}\left(\dot{x}_{\mathrm{B}}-\dot{x}_{\mathrm{A}}\right)-F_{\mathrm{fA}}-F_{\mathrm{PA}} \\
m_{\mathrm{B}} \ddot{x}_{\mathrm{B}}=-k_{P}\left(x_{\mathrm{B}}-x_{\mathrm{A}}\right)-c_{p}\left(\dot{x}_{\mathrm{B}}-\dot{x}_{\mathrm{A}}\right)-F_{\mathrm{fB}}+F_{\mathrm{PB}} \\
F_{\mathrm{PA}}=\frac{1}{3} m_{\mathrm{PA}} a_{\mathrm{A}}+F_{P}=\frac{1}{3}\left(\frac{\dot{x}_{\mathrm{A}}}{\dot{x}_{\mathrm{A}}-\dot{x}_{\mathrm{B}}}\right) m_{P} \ddot{x}_{\mathrm{A}}+F_{P} \\
F_{\mathrm{PB}}=-\frac{1}{3} m_{\mathrm{PB}} a_{\mathrm{B}}+F_{P}=\frac{1}{3}\left(\frac{\dot{x}_{\mathrm{B}}}{\dot{x}_{\mathrm{A}}-\dot{x}_{\mathrm{B}}}\right) m_{P} \ddot{x}_{\mathrm{B}}+F_{P} \\
F_{\mathrm{fA}}=\left\{F_{\mathrm{CA}} \lambda\left(\dot{x}_{\mathrm{A}}\right)+F_{\mathrm{SA}}\left[1-\lambda\left(\dot{x}_{\mathrm{A}}\right)\right]\right\} \operatorname{sign}\left(\dot{x}_{\mathrm{A}}\right) \\
F_{\mathrm{fB}}=\left\{F_{\mathrm{CB}} \lambda\left(\dot{x}_{\mathrm{B}}\right)+F_{\mathrm{SB}}\left[1-\lambda\left(\dot{x}_{\mathrm{B}}\right)\right]\right\} \operatorname{sign}\left(\dot{x}_{\mathrm{B}}\right)
\end{array}\right.
$$

Further, in the above equations, the PA driving force is $F_{P}=k_{P} d_{e} V$, where $k_{P}$ denotes the mechanical stiffness and $d_{e}$ denotes piezoelectric coefficient (Fung et al., 2008; Low and Guo, 1995). In our case, $k_{P}=5 \times 10^{7} \mathrm{~N} \mathrm{~m}^{-1}$, $d_{e}=3 \times 10^{-7} \mathrm{~m} \mathrm{~V}^{-1}, m_{P}=120 \mathrm{~g}$. In the test system, there is a support force from the pipe acting on the block against gravity. Consequently, the block gets a friction force $F_{\mathrm{SB}}=0.1 \mathrm{~N}$, which is however much smaller than $F_{\mathrm{SA}}$. So $F_{\mathrm{CB}} \approx 0$ while $F_{\mathrm{CA}}=5 / 6 F_{\mathrm{SA}}$. The waveform of the input voltage has a strong influence on the performance of the IDM robot. A triangular waveform was employed in this study to drive the PA. As shown in Fig. 4, the duty ratio was 75 and $80 \%$, respectively, in this study. Duty ratio is defined as the period of time from zero to peak $\left(t_{1}\right)$ of the wave over the total period of operational time $\left(t_{2}\right)$, namely $t_{1} / t_{2}$.

Figure 5 shows the displacements when the driving voltage is $80 \mathrm{v}, t_{1}=1.5 \mathrm{~ms}$, and $t_{2}=2 \mathrm{~ms}$. The Object A moves backward a little when the PA extends slowly. The actuator pulls Object A forward during its quick contraction. 


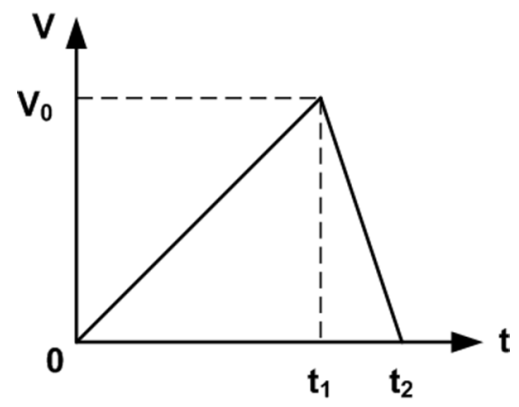

Figure 4. The waveform of the input voltage. The duty ratio is defined as $t_{1} / t_{2}$ and it is 75 and $80 \%$, respectively, in this study; $V_{0}$ is the maximum voltage.

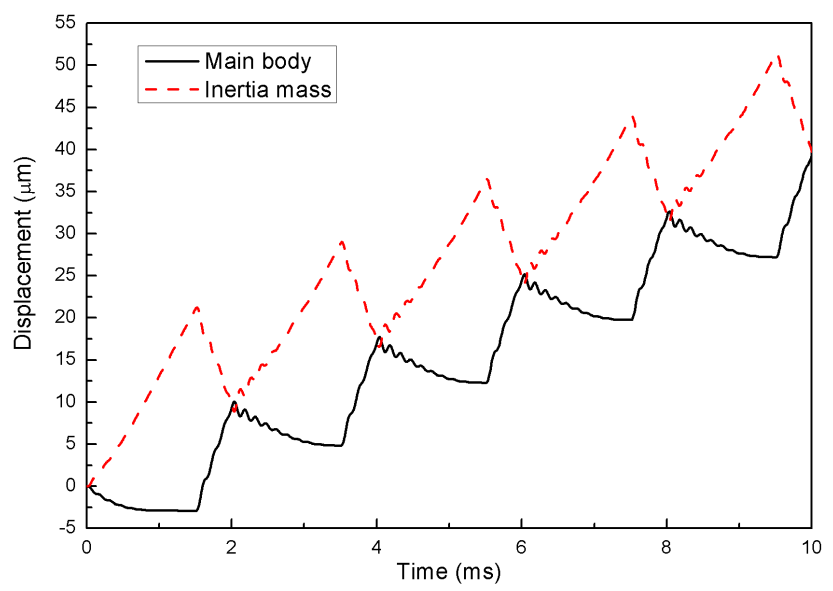

Figure 5. Displacements of Object A and block in 5 cycles.

\subsection{Model validation}

An experimental validation has been carried out on an optical stage. Figure 6 is a schematic diagram of the test-bed. The robot was driven by a generator and an amplifier (PILVPZT). The inner wall of the pipe is made of stainless steel with the radius of $18 \mathrm{~mm}$ in Fig. 7. The movement of the robot has been measured by a laser triangulation sensor (Keyence ${ }^{\mathrm{TM}}$ LKH008).

Figure 8 shows the velocity of the main body (Object A). It can be seen that the predicted result with the developed model is closer to the measurement than to the model without consideration of the inertia of PA. Further, both models are able to improve the prediction accuracy with the voltage being less than $30 \mathrm{v}$. This is reasonable, when the input voltage is small, the kinetic energy of the robot is small, and the effect of the inertia is small accordingly. After the voltage is greater than $30 \mathrm{v}$, the difference between the two models tends to increase with respect to the measured result (i.e. the error of the model without consideration of the inertia of the PA increases) with the increase of the voltage.

Figure 9 shows the result of the velocity with driving frequency. The driving voltage is $80 \mathrm{v}$, and the duty ratio is

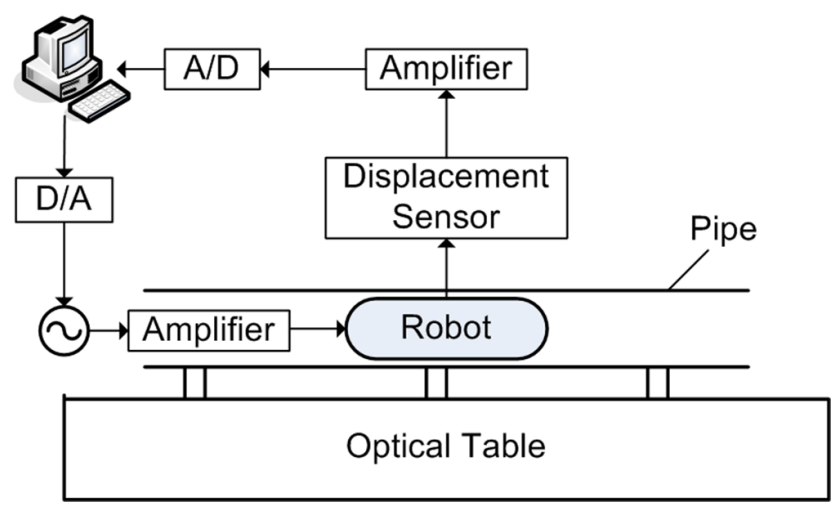

Figure 6. The schematic diagram of the signal flow.

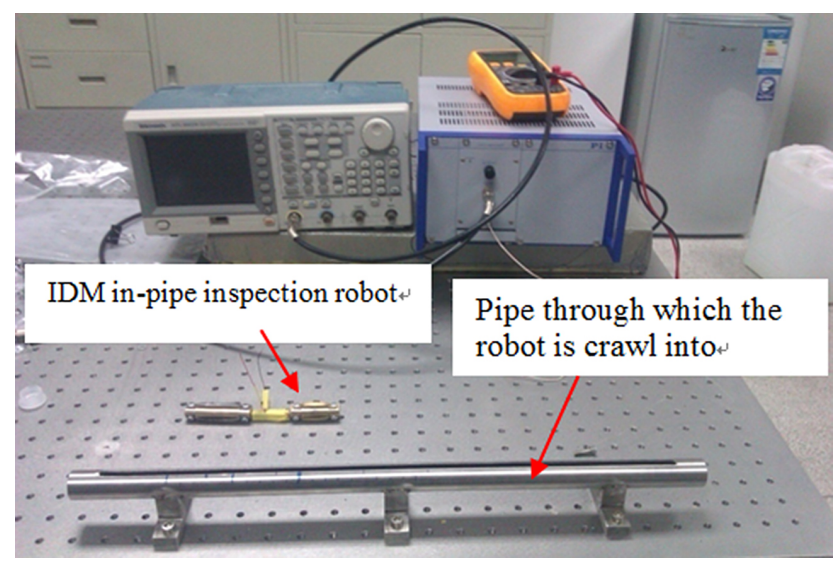

Figure 7. The robot and physical set up for testing.

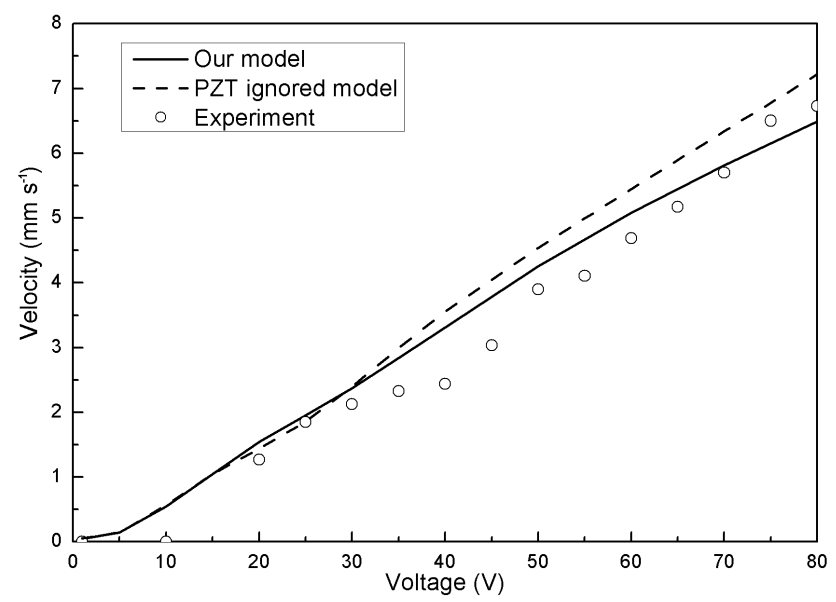

Figure 8. Velocity vs. voltage.

$75 \%$. There are two main peaks of the velocity. It is noted that the frequencies are employed as the excitation signals to examine system behavior in terms of energy efficiency. It can be found that the measured locations of the peak values agree well with the one predicted by the models. At the 


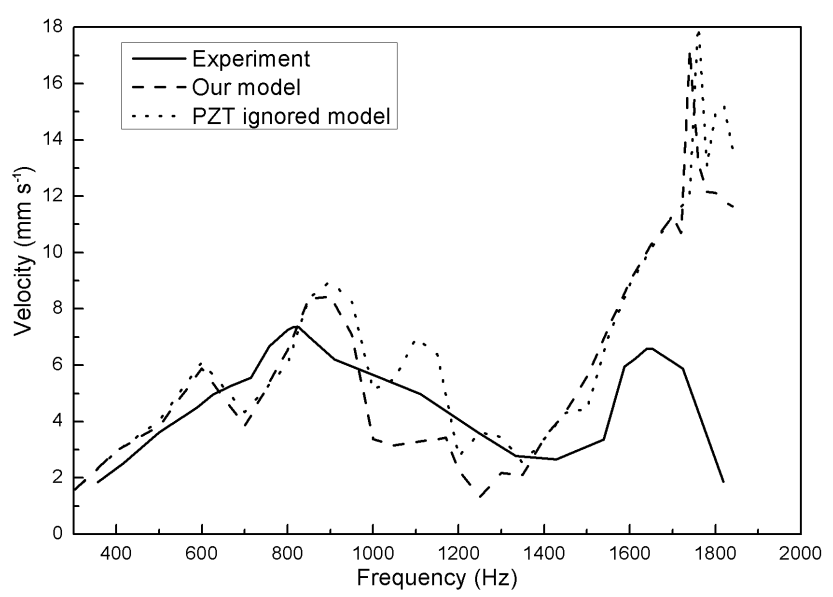

Figure 9. The velocity vs. frequency.

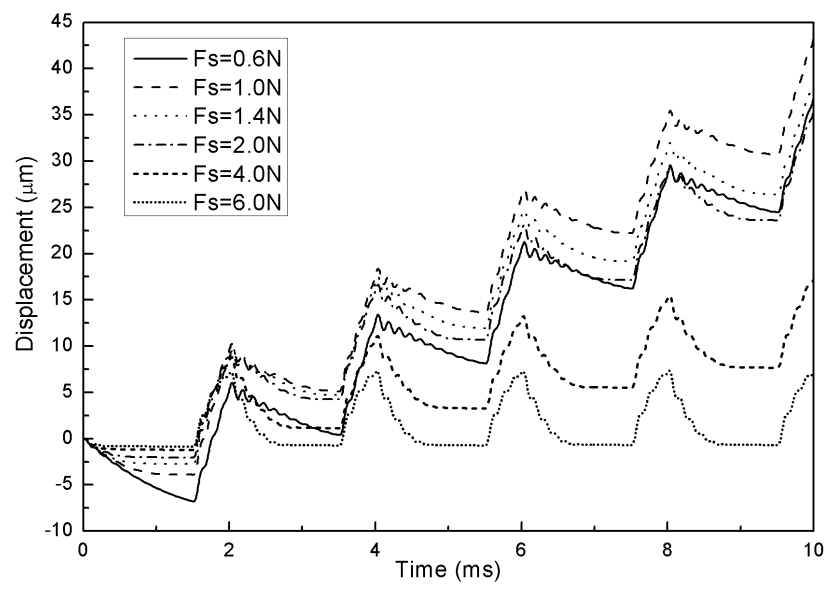

Figure 10. Displacements of the main body versus different friction coefficients.

second peak value, the one predicted by the models is much larger than the measured one. This is because under a high frequency excitation, the mechanical loss and heat consumption are significant in the real structure, while this feature has not been considered in the model. Non-uniform friction along the pipe is also responsible for the velocity versus frequency relation. Nevertheless, the developed model is better than the model without consideration of the inertia of the PA.

Overall, the error of the model with consideration of the inertia of the PA is $8.65 \%$ while the error of the model without consideration of the inertia of the PA is $14.34 \%$, with respect to the measured result. In the experiment, the measurement approach has introduced a relatively large error which is up to about $10 \%$ (the displacement measurement is based on the ruler with the resolution of $0.1 \mathrm{~mm}$, which translate to an error of $10 \%$ ). As such, the developed model shows an acceptable accuracy.

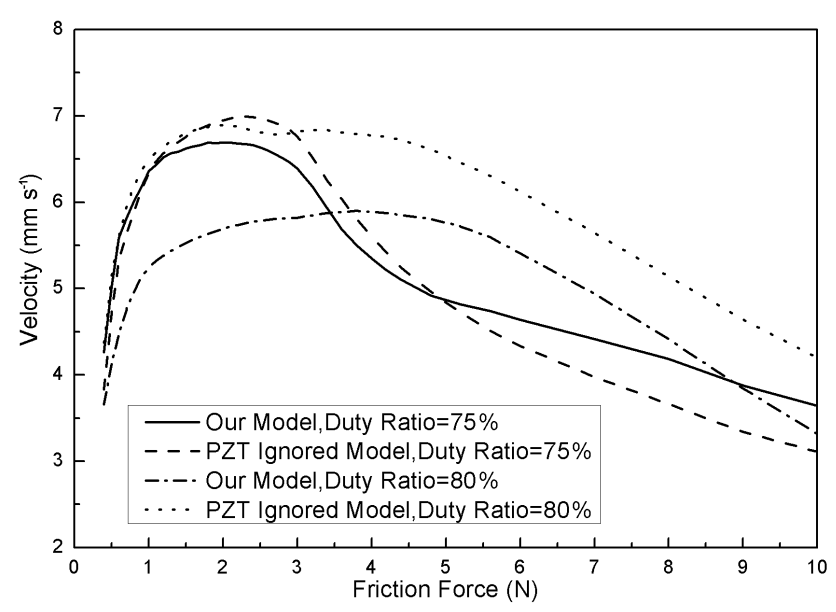

Figure 11. Velocity of the robot versus friction.

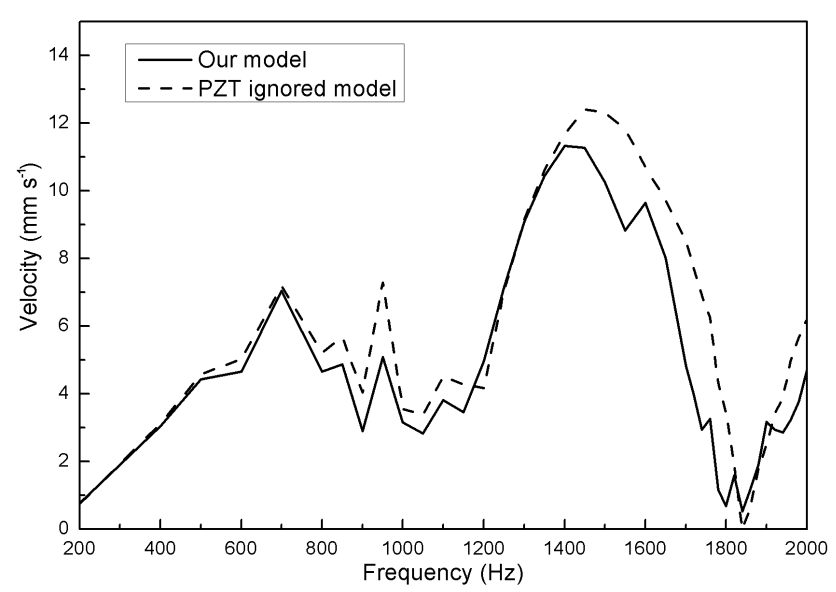

Figure 12. The velocity of the actuator versus the frequency of the input voltage.

\section{Notes on optimization of the performance of the in-pipe inspection robot}

Figure 10 shows the displacement of the main body under different frictions. Too small or too large friction will cause the failure of the robot. The largest step movement happens when the friction force is around $1.0 \mathrm{~N}$. As the friction force reaches $6 \mathrm{~N}$, the robot does not move forward in one cycle at all, which implies a failure. Figure 11 shows the velocity of the robot with respect to the friction. It is shown that an optimal velocity can be reached by adjusting the friction force.

Figure 12 shows the velocity with respect to the frequency. It has many velocity peaks from the frequency range from 200 to $2000 \mathrm{~Hz}$. The frequencies at the peaks of velocity are slightly different between two models. When the frequency is high, the velocity predicted with our model is smaller than the model without consideration of the inertia of the PA. This is so because the mass of the PA also consume the driving energy. The dynamic behaviors are quite different when the 
mass of the PZT actuator is taken into consideration, such as response displacement, resonant frequency. These factors are crucial when we consider the control strategy.

\section{Conclusions}

This paper presented a work towards the development of a model that can capture the continuous inertia of the PA in IDM in the context of pipe inspection and has a balanced accuracy and computational efficiency. The work also included an experimental study to analyze the effectiveness of the model. It can be concluded from this study that the developed model can predict the system behavior significantly more accurate than the model without consideration of the inertia of the PA. Since the model is also computationally efficient, it has a high potential to be used for feedback control of the system as well as design optimization of the system.

Acknowledgements. This work was supported in part by the National Natural Science Foundation of China (Grant No. 51305138 and 51375166), the Fundamental Research Funds for the Central Universities and Science and Technology Commission of Shanghai Municipality (Grant No. 13ZR1453300).

Edited by: G. Hao

Reviewed by: Q. Xu and one anonymous referee

\section{References}

Chang, S. H. and Li, S. S.: A High Resolution Long Travel Frictiondrive Micro-positioner with Programmable Step Size, Rev. Sci. Instrum., 70, 2776-2782, 1999.

Dario, P., Hannaford, B., and Menciassi, A.: Smart surgical tools and augmenting devices, IEEE Trans. Robot. Autom., 19, 782791, 2003.

Fatikow, S. and Rembold, U.: Microsystem Technology and Microrobotics, Springer-Verlag, Berlin, Heidelberg, 303-361, 2009.

Fukui, R., Torii, A., and Ueda, A.: Micro robot actuated by rapid deformation of piezoelectric elements, International Symposium on Micromechatronics and Human Science, 9-12 September 2001, Nagoya, Japan, 117-122, 2001.

Fung, R. F., Han, C. F., and Ha, G. L.: Dynamic Responses of the Impact Drive Mechanism Modeled by the Distributed Parameter System, Appl. Math. Model., 32, 1734-1743, 2008.

Ha, J. L., Fung, R. F., and Han, C. F.: Optimization of an impact drive mechanism based on real-coded genetic algorithm, Sensors Actuat. A, 121, 488-493, 2005.

Hattori, S., Hara, M., Nabae, H., Hwang, D., and Higuchi, T.: Design of an impact drive actuator using a shape memory alloy wire, Sensors Actuat. A, 219, 45-47, 2014.

Higuchi, T., Furutani, K., Yamagata, Y., Kudoh, K., and Ogawa, K.: Improvement of velocity of impact drive mechanism by controlling friction, J. Adv. Automat. Tech., 5, 71-76, 1993.
Hunstig, M., Hemsel, T., and Sextro, W.: Stick-slip and slip-slip operation of piezoelectric inertia drives. Part I: Ideal excitation, Sensors Actuat. A, 200, 90-100, 2013.

Karnopp, D.: Computer Simulation of Stick-slip in Mechanical Dynamic System, J. Dyn. Syst.-ASME, 107, 100-103, 1985.

Li, J. W., Zhang, W. J., Yang, G. S., Tu, S. D., and Chen, X. B.: Thermal-error modeling for complex physical systems the-stateof-arts review, Int. J. Adv. Manufact. Technol., 42, 168-179, 2009.

Li, Q., Zhang, W. J., and Chen, L.: Design for control (DFC): a concurrent engineering approach for mechatronic system design, IEEE-ASME T. Mech., 6, 161-169, 2001.

Liu, P. K., Wen, Z. J., and Sun, L. N.: An in-pipe micro robot actuated by piezoelectric bimorphs, Chinese Sci. Bull., 54, 21342142, 2009.

Liu, Y. F., Li, J., Hu, X. H., Zhang, Z. M., Cheng, L., Lin, Y., and Zhang, W. J.: Modeling and control of piezoelectric inertiafriction actuators: review and future research directions, Mech. Sci., 6, 95-107, doi:10.5194/ms-6-95-2015, 2015.

Low, T. S. and Guo, W.: Modeling of Three-layer Piezoelectric Bimorph Beam with hysteresis, IEEE-ASME J. Microelectromech. S., 4, 230-237, 1995.

Makkar, C., Hu, G., Sawyer, W. G., and Dixon, W. E.: LyapunovBased Tracking Control in the Presence of Uncertain Nonlinear Parameterizable Friction, IEEE T. Automat. Contr., 52, 19881994, 2007.

Ouyang, P. R., Tjiptoprodjo, R. C., Zhang, W. J., and Yang, G. S.: Micro-motion Devices Technology: The State of Arts Review, Int. J. Adv. Manufact. Techol., 38, 463-478, 2008.

Sabzehmeidani, Y., Mailah, M., and Hussein, M.: Intelligent Control and Modelling of a Micro robot for in-pipe application, ECME, Puerto De La Cruz, Spain, p. 30, 2010.

Tenzer, P. E. and Mrad, R. B.: A systematic procedure for the design of piezoelectric inchworm precision positioners, IEEE-ASME T. Mech., 9, 427-435, 2004.

Xia, Q. X., Xie, S. W., and Huo, Y. L.: Numerical simulation and experimental research on the multi-pass neck-spinning of nonaxisymmetric offset tube, J. Mater. Process. Tech., 206, 500-508, 2008.

Yokose, T., Hosaka, H., Yoshida, R., and Morita, T.: Resonance frequency ratio control with an additional inductor for a miniaturized resonant-type SIDM actuator, Sensors Actuat. A, 214, 142$148,2014$.

Yokozawa, H. and Morita, T.: Wireguide driving actuator using resonant-type smooth impact drive mechanism, Sensors Actuat. A, 230, 40-44, 2015.

Yukawa, T., Suzuki, M., and Satoh, Y.: Design of magnetic wheels in-pipe inspection robot, IEEE-ASME J. Microelectromech. S., 15, 1289-1298, 2006.

Zhang, W. J., Li, Q., and Guo, S. L.: Integrated Design of Mechanical Structure and Control Algorithm for a Programmable FourBar Linkage, IEEE-ASME T. Mech., 4, 354-362, 1999.

Zhang, Z. M., An, Q., Li, J. W., and Zhang, W. J.: Piezoelectric friction-inertia actuator - a critical review and future perspective, Int. J. Adv. Manuf. Tech., 62, 669-685, 2012. 